\title{
I. On the origin of nebulæ
}

\author{
James Croll LL.D. F.R.S.
}

To cite this article: James Croll LL.D. F.R.S. (1878) I. On the origin of nebulæ, Philosophical Magazine Series 5, 6:34, 1-14, DOI: 10.1080/14786447808639464

To link to this article: http://dx.doi.org/10.1080/14786447808639464

$$
\text { 曲 Published online: } 13 \text { May } 2009 .
$$

Submit your article to this journal 전

LII Article views: 3

Q View related articles ¿ 
LONDON, EDINBURGH, AND DUBLIN

\title{
PHILOSOPHICAL MAGAZINE
}

\author{
AND \\ JOURNAL OF SCIENCE.
}

[FIFTH SERIES.]

$J U L Y 1878$.

\section{On the Origin of Nebula. By JaMes CroLl, LL.D., F.R.S.*}

THE object of the present communication is to examine 1 the bearings of the modem science of energy on the question of the origin of nebula, and in particular to consider the physical cause of the dispersion of matter into stellar space in the nebulous form. In doing so I have studiously avoided the introduction of mere hypotheses and principles not generally admitted by physicists. These remarks may be necessary, as the title of the paper might otherwise lead to the belief that it is on a speculative subject lying outside the province of the physicist.

The question of the origin of nebulæ is simplified by the theory, now generally received, that stars are suns like our own, and that nebulæe are in all probability stars in process of formation. The problem will therefore be most readily attacked by considering, first, the origin of our sun, as this orb, being the one most accessible to us, is that with which we are best acquainted.

By the origin of the sun I do not, of course, mean the origin of the matter constituting the sun-this being an inquiry with which the physicist has nothing whatever to do-but simply its origin as a sun, $i . e$. as a source of light and heat. Our first question must therefore be, What is the origin of the sun's heat? From what source did he derive that enormous amount of energy which in the form of heat he has been dis-

* Communicated by the Author.

Phil. .Mag. S. 5. Vol. 6. No. 34. July 1878. 
sipating into space during past ages? Difficult as the question at first sight appears to be, it is yet simplified and brought within very narrow limits when we remember that there are only two conceivable sources. The sun must have derived his energy either from Gravitation, or from that other source to which I directed attention several years ago*, Motion in Space. All other sources of energy put together could not have supplied our luminary with one thousandth part of that which he has possessed. We are therefore compelled to attribute the sun's heat to one or other of these two, or to give up the whole inquiry as utterly hopeless. The important difference between the two is that the store of energy derivable from Gravitation could not possibly have exceeded 20 to 30 million years' supply of heat at the present rate of radiation; whereas the store derivable from Motion in Space, depending on the rate of that motion, may conceivably have amounted to any assignable quantity. Thus a mass equal to that of the sun, moving with a velocity of 476 miles per second, possesses in virtue of that motion energy sufficient, if converted into heat, to cover the present rate of the sun's radiation for 50 million years. Twice that velocity would give 200 million years' heat ; four times that velocity would give 800 million years' heat, and so on without limit.

It is, however, not enough that we should have in the form of motion in space energy sufficient. We must have a means of converting this motion into heat-of converting motion of translation into molecular motion. To understand how this can be effected, we simply require the conception of Collision. Two bodies moving towards each other will have their motion of translation converted into molecular motion (heat) by their encounter.

To which of these two causes must we attribute the Sun's heat? It is certain that gravitation must have been a cause ; and if we adopt the nebular hypothesis of the origin of our solar system, then from 20 to 30 million years' heat may thus be accounted for. But we know from geological evidence that the sun has been dissipating his light and heat at about the present rate for a much longer period. In a paper published in the 'Quarterly Journal of Science' for July 1877, I have discussed the geological evidence for the age of the earth at considerable length, and have pointed out that the time which has elapsed since life began on the globe cannot have been less than 60 million years. This estimate is based upon a rough estimate of the thickness of rock which has been removed by subaerial denudation since the earliest epoch of which geolo-

* Phil. Mag. May 1868. 
gists take cognizance. Measuring the rate of the subaerial denudation by a method which I pointed out several years ago*, we are able to determine roughly the time required for the removal of the rock. But the 60 million years thus obtained, be it observed, are only the inferior limit. We know that a certain amount of rock has been removed; but how much more may have been carried away we cannot tell. Consequently, although we have good grounds for believing that 60 million years have elapsed since life began on the globe, yet the lapse of time may really have been very much longer. We are justified, therefore, in concluding that our globe has been receiving from the sun for the past 60 million years an amount of light and heat daily not very sensibly less than at present. This shows that gravitation alone will not explain the origin of the sun's heat, and that a far more effective cause must be found. Now the only other conceivable cause exceeding that of gravity is, of course, motion in space.

If the gravitation theory fails to explain the origin of the sun, it fails yet more decidedly to account for the nebulæ. In fact it does not attempt any explanation of the origin of the latter ; for it begins by assuming their existence, and not only so, but that they are in process of condensation. This must be the case, because the theory in question assumes that the particles of a nebulous mass have, in virtue of gravity, a mutual tendency to approach one another; and it cannot tell us how this tendency could exist without producing its effect. The advocates of the theory are not at liberty to call in the aid of heat in order to explain why the particles are not mutally approaching; because it is this mutual approach which, according to the theory, produces the heat, and of conrse without such approach no heat could be generated. A nebulous mass with a tendency to condensation could not have existed from eternity as such; but what the previous condition of a nebula was, and how it came to assume its present state, the gravitation theory cannot say. It begins with a star or sun in process of formation, but does not help us to understand how the process of formation commenced.

It is quite otherwise, however, with the other theory. This latter does not, like the former, begin by assuming the existence of a nebulous mass; on the contrary, it goes back to the very commencement of physical inquiry, to the very point where physical investigation takes its rise, and beyond which we cannot penetrate. The only assumption it makes is that of the existence of matter and motion-if indeed this can be called an assumption. How matter and motion began to be,

* Phil. Mag. May 1868, and February 1867. 
whether they were eternal or were created, are questions wholly beyond the domain of the physicist. The theory takes for a fact the existence of stellar masses in a state of motion; and its advocate is not required, as a physicist, to account for the existence either of those masses or of their motions. Neither is it necessary for him to advance any hypothesis to show how the masses came into collision; for unless we are to assume that all stellar masses are moving in one direction and with uniform velocity (a supposition contrary to known facts), then collisions must occasionally take place. The chances are that stellar masses are of all sizes, moving at random in all directions and with all velocities. We have here therefore, without any hypothesis, all the conditions necessary for the origin of nebuli. Take the case of the origin of the nebulous mass out of which our sun is believed to have been formed. Suppose two bodies, each one half the mass of the sum, approaching each other directly at the rate of 476 miles per second (and there is nothing at all improbable in such a supposition), their collision would transform the whole of the motion into heat affording an amount sufficient to supply the present rate of radiation for 50 million years. Each pound of the mass would, by the stoppage of the motion, possess not less than $100,000,000,000$ foot-pounds of energy transformed into heat, or as much heat as would suffice to melt 90 tons of iron or raise 264,000 tons $1^{\circ} \mathrm{C}$. The whole mass would be converted into an incandescent gas, with a temperature of which we can form no adequate conception. If we assume the specific heat of the gaseous mass to be equal to that of air (viz. 2374 ), the mass would have a temperature of about $300,000,000^{\circ} \mathrm{C}$., or more than 140,000 times that of the voltaic are.

Reason why Nebulce occupy so much Space.-It may be objected that enormous as would be such a temperature, it would nevertheless be insufficient to expand the mass against gravity so as to occupy the entire space included within the orbit of Neptune. To this objection it might be replied, that if the temperature in question were not sufficient to produce the required expansion, it might readily have been so if the two bodies before encounter be assumed to possess a higher velocity, which of course might have been the case. But without making any such assumption, the necessary expansion of the mass can be accounted for on very simple principles. It follows in fact from the theory, that the expansion of the gaseous mass must have been far greater than could have resulted simply from the temperature produced by the concussion. This will be obvious by considering what must take place immediately after the encounter of the two bodies, 
and before the mass has had sufficient time to pass completely into the gaseous condition. The two bodies coming into collision with such enormous velocities would not rebound like two elastic balls, neither would they instantly be converted into vapour by the encounter. The first effect of the blow would be to shiver them into fragments, small indeed as compared with the size of the bodies themselves, but still into what might be called in ordinary language immense blocks. Before the motion of the two bodies conld be stopped, they would undoubtedly interpenetrate each other; and this of course would break them up into fragments. But this would only be the work of a few minutes. Here, then, we should have all the energy of the lost motion existing in these blocks as heat (molecular motion), while they were still in the solid state; for as yet they would not have had sufficient time to assume the gaseous condition. It is obvious, however, that the greater part of the heat would exist on the surface of the blocks (the place receiving the greatest concussion), and would continue there while the blocks retained their solid condition. It is difficult in imagination to realize what the temperature of the surfaces would be at this moment. For, supposing the heat were uniformly distributed through the entire mass, each pound, as we have already seen, would possess $100,000,000,000$ foot-pounds of heat. But as the greater part of the heat would at this instant be concentrated on the outer layers of the blocks, these layers would be at once transformed into the gaseous condition, thus enveloping the blocks and filling the interspaces. The temperature of the incandescent gas, owing to this enormous concentration of heat, would be excessive, and its expansive force inconceivably great. As a consequence the blocks would be separated from each other, and driven in all directions with a velocity far more than sufficient to carry them to an infinite distance against the force of gravity were no opposing obstacle in their way. The blocks by their mutual impact would be shivered into smaller fragments, each of which would consequently become enveloped in incandescent gas. These smaller fragments would in a similar manner break up into still smaller pieces, and so on until the whole came to assume the gaseous state. The general effect of the explosion, however, would be to disperse the blocks in all directions, radiating from the centre of the mass. Those towards the outer circumference of the mass, meeting with little or no obstruction to their onward progress, would pass outwards into space to indefinite distances, leaving in this manner a free path for the layers of blocks behind them to follow in their track. Thus eventually a space, perhaps twice or even thrice that in- 
cluded within the orbit of Neptune, might be filled with fragments by the time the whole had assumed the gaseous condition.

It would be the suddenness and almost instantaneity with which the mass would receive the entire store of energy, before it had time even to assume the molten, far less the gaseous condition, which would lead to such fearful explosions and dispersion of the materials. If the heat had been gradually applied, no explosions, and consequently no dispersion, of the materials would have taken place. There would first have been a gradual melting; and then the mass would pass by slow degrees into vapour, after which the vapour would rise in temperature as the heat continued until it became possessed of the entire amount. But the space thus occupied by the gaseous mass would necessarily be very much smaller than in the case we have been considering, where the shattered materials were first dispersed into space before the gaseous condition was assumed.

Reason why Nebulce are of such various Shapes.-The latter theory accounts also for the various and irregular shapes assumed by the nebulæ; for although the dispersion of the materials would be in all directions, it would, according to the law of chances, very rarely take place uniformly in all directions. There would generally be a greater amount of dispersion in certain directions, and the materials would thus be carried along various lines and to diverse distances; and although gravity would tend to bring the widely scattered materials ultimately together into one or more spherical masses, yet, owing to the exceedingly rarefied condition of the gaseous mass, the nebula would change form but slowly.

Reason why Nebule emit such Feelle Light.-The feeble light emitted by nebulæ follows as a necessary result from the theory. The light of nebulæ is mainly derived from glowing hydrogen and nitrogen in a gaseous condition; and it is well known that these gases are exceedingly bad radiators. The oxyhydrogen flame, though its temperature is only surpassed by that of the voltaic are, gives nevertheless a light so feeble as scarcely to be visible in the daytime. Now, even supposing the enornous space occupied by a nebula were due to excessive temperature, the light emitted would yet not be intense were it derived from nitrogen or hydrogen gas. The small luminosity of nebulæ, however, is due to a different cause. The enormous space occupied by those nebulx is not so much owing to the heat which they possess, as to the fact that their materials were dispersed into space before they had time to pass into the gaseous conrition; so that, by the time this latter state was assumed, the 
space occupied was far greater than was demanded either by the temperature or the amount of heat received.

If we adopt the nebular hypothesis of the origin of our solar system, we must assume that our sun's mass, when in the condition of nebula, extended beyond the orbit of the planet Neptune, and consequently filled the entire space included within that orbit. Supposing Neptune's orbit to have been its outer limit, which it evidently was not, it would nevertheless have then occupied $274,000,000,000$ times the space that it does at present. We shall assume, as before, that 50 million years' heat was generated by the concussion. Of course there might have been twice or even ten times that quantity; but it is of no importance what number of years is in the meantime adopted. Enormous as 50 million of years' heat is, it yet gives, as we shall presently see, only 32 foot-pounds for each cubic foot. The amount of heat due to concussion being equal, as before stated, to $100,000,000,000$ foot-pounds for each pound of the mass, and a cubic foot of the sun at his present density of 1.43 weighing 89 lbs., each cubic foot must have possessed 8,900,000,000,000 foot-pounds. But when the mass was expanded to occupy $274,000,000,000$ times more space, which it would do when it extended to the orbit of Neptune, the heat possessed by each cubic foot would then amount to only 32 foot-pounds.

In point of fact, however, it would not even amount to that; for a quantity equal to upwards of 20 million years' heat would necessarily be consumed in work against gravity in the expansion of the mass; all of which would, of course, be given back in the form of heat as the mass contracted. During the nebulous condition it would not exist as heat, so that only 19 foot-pounds out of the 32 foot-pounds generated by concussion would then exist as heat. The density of the nebula would be only $\frac{1}{16248160}$ that of hydrogen at ordinary temperature and pressure. The 19 foot-pounds of heat in each cubic foot would nevertheless be sufficient to maintain an excessive temperature; for there would be in each cubic foot only $\frac{1}{440000}$ of a grain of matter. But although the temperature would bo excessive, the quantity both of light and heat in each cubic foot would of necessity be small. The heat being only $\frac{1}{71}$ of a thermal unit, the light emitted would certainly be exceedingly feeble, resembling very much the electric light in a vacuum-tube.

Heat and Light of Nebulce cannot result from Condensation.The fact that nebula are not only self-luminous but indicate the existence of hydrogen and nitrogen in an incandescent condition proves that they must possess a considerable tempe- 
rature. And it is scarcely conceivable that the temperature could have been derived from the condensation of their masses. When our sun was in the nebulous condition it no doubt was self-luminous like other nebulæ, and doubtless would have appeared, if seen from one of the fixed stars, pretty much like other nebula as viewed from our earth. The spectrum would no doubt have revealed in it the presence of incandescent gas. At all events we have no reason to conclude that our nobula was in this respect an exception to the general rule, and essentially different from others of the same class. The heat which our nebula could have derived from condensation up to the time that Neptune was formed, no matter how far the outer circumference of the mass may originally have extended beyond the orbit of that planet, could not have amounted to over $\frac{1}{7000000}$ of a thermal unit for each cubic foot; and the quantity of light given out could not possibly have rendered the mass visible. Consequently the heat and light possessed by the mass must have been derived from some other source than that of gravity.

We have further evidence that the heat and light of nebulæ cannot have been derived from condensation. If there be any truth, as there doubtless is, in Mr. Lockyer's view of the evolution of the planets, then the nebulæ out of which these bodies were evolved must have originally possessed a very high temperature - a temperature so high, indeed, as to produce perfect chemical dissociation of the elements. In short, "the temperature of the nebulæ," as Mr. Lockyer remarks *, "was then as great as the temperature of the sun is now." Mr. Lockyer's theory is that the metals and the metalloids, owing to excessive temperature, existed in the nebulous mass uncombined-the metals, owing to their greater density, assuming the central position, and the metalloids keeping to the outside. The denser the metal the nearer would its position be to the centre of the mass, and the lighter the metalloid the nearer to the outside. As a general rule the dissociated elements would arrange themselves according to their densities; and it is for this reason, be considers, that the outer planets Neptune, Uranus, Saturn, and Jupiter, are less dense than the inner planets, since they must have been formed chiefly of metalloids, while the inner and more dense planets would consist chiefly of metallic elements.

"The hypothesis," says Mr. Lockyer, "is almost worthless unless we assume very high temperatures, because unless you have heat enough to give perfect dissociation, you will not have that sorting-out which always seems to follow the same

" "Why the Larth's Chemistry is as it in,".p. 55, 1877. 
law." But the heat which produced this dissociation previous to the formation of the planets could not have been derived from the condensation of the nebula ; for the quantity so derived prior to the existence of the outermost planet must have been infinitesimal indeed. The heat existing in the nebula previous to condensation must have come from some source; and we can conceive of no other save that which we have been considering.

The Gaseous State the first Condition of a Nebula.-If the foregoing be the true explanation of the origin of nebulæ, it will follow that the gaseous state will in most cases be the first or original condition, and that a nebula giving a continuous spectrum will only be found after it has condensed to a considerable extent.

The irresolvable nebulæ which exhibit bright lines, in all probability consist, as Mr. Huggins maintains, of glowing gas without any thing solid in them. In short they are nebulæ in their first stage of development, and have not as yet condensed sufficiently to become possessed of nuclei. If we adopt the generally accepted nebular hypothesis, I cannot understand how we can consistently deny the existence of gaseous nebulæ; for, according to the nebular hypothesis, the central nucleus which constitutes a sun or star, and which exhibits a continuous spectrum, was formed by condensation as surely as the planets or the satellites have been. Were we to go back sufficiently far in the past, we should come to a time when not only our globe but the sun himself consisted of gaseous matter only. If we admit this, then why not also admit that there may be nebulæ at the present time in a condition similar to what our sun must formerly have been.

The gaseous condition of nebulæ seems to follow as a consequence from Mr. Lockyer's theory. For in order that the materials in the formation of a sun or star may arrange themselves according to their densities, dissociation is requisite; but there can be no dissociation except in the gaseous condition.

Star-Clusters.-The wide-spread and irregular manner in which the materials would in many cases be distributed through space after collision, would prevent a nebula from condensing into a single mass. Subordinate centres of attraction, as was long ago shown by Sir William Herschel (in his famous memoir on the formation of stars*), would be established, around which the gaseous particles wonld arrange themselves and gradually condense into separate stars, which would finally assume the condition of a cluster.

* Phil. Trans, for 1811. 
Binary, Triple, and Multiple systems of stars will of course be accounted for in a similar manner.

It is conceivable that it may sometimes happen that by the time the materials are broken up and dissipated into space, there may not be sufficient heat left to convert the fragments into vapour. In this ease we should have what Professor Tait has suggested, a nebula consisting of "clouds of stones." But such nebulæ must be of rare occurrence.

Objections considered.-On a former occasion I considered one or two anticipated objections to the theory that stellar light and heat were derived from motion in space. But as these objections have since been repeatedly urged by physicists both in this country and in America, I shall again briefly refer to them.

Objection 1st. "The existence of such non-luminous bodies as the theory assumes is purely conjectural, as no such bodies have ever been observed." In reply, it is just as legitimate an inference that there are bodies in stellar space not luminous as that there are luminous bodies in space not visible. We have just as good evidence for believing in the existence of the one as we have in the existence of the other. Bodies in stellar space can only be known through the eye to exist. If they are not luminous, they of course cannot be seen. But we are not warranted on that account to suppose that they do not exist, any more than we have to suppose that stars do not exist which are beyond the reach of our vision. We have, however, positive evidence that there are bodies in space nonluminous, as the meteorites and planets for example. The stars are beyond doubt suns like our own; and we cannot avoid the inference that, like our sun, they are surrounded by planets. If so, then we have to admit that there are far more bodies in stellar space non-luminous than luminous. But this is not all: the stars no more than our sun can have been dissipating their light and heat during all past ages ; their light and heat must have had a beginning; and before that they could not be luminous. Neither can they continue to give out light and heat eternally; consequently when their store of energy is exhausted they will be non-luminous again. light and heat are not the permanent possession of a body. A body may retain its energy in the form of motion undiminished and untransformed through all eternity, but not so in the form of heat and light. These are forms of energy which are being constantly dissipated into space and lost in so far as the body is concerned.

The conclusion to which we are therefore led is that there are in all probability bodies in stellar space which have not 
yet received their store of light and heat, while there are others which have entirely lost it. The stars are probably only those stellar masses which having recently had an encounter have become possessed of light and heat. They have gained in light and heat what they have lost in motion, but they have gained a possession which they cannot retain, and when it is lost they become again what they originally were $\longrightarrow$ dark bodies.

2nd. "We have no instances of stellar motions comparable with those demanded by the theory." A little consideration will show that this is an objection which, like the former, can hardly be admitted. No body of course moving at the rate of 400 miles per second could remain a member of our solar system; and beyond our system the only bodies visible are the nebula and fixed stars; and they are according to the theory visible because like the sun they have lost their motion-the lost motion being the origin of their light and heat. Their comparatively small velocities are in reality evidence in favour of the theory than otherwise; for had the stars been moving with excessive velocities this would have been adduced as proof that their light and heat could not have been derived from motion lost, as the theory assumes.

3rd. "If suns or stars have been formed by collision of bodies moving in space, proper motion can be none other than the unused and unconverted energy of the original components. And as stellar bodies are likely of all sizes and moving with all manner of velocities, it must often happen, from the unequal force of the impinging masses, that a large proportion of the original motion must remain unconverted into heat. Consequently some of the stars ought, according to the theory, to possess great velocities-which is not the case, as none of the stars have a motion of more than 30 or 40 miles per second."

I freely admit that, if it could be proved that none of the stars have a proper motion of more than 30 or 40 miles per second, it would at least be a formidable difficulty in the way of accepting the theory. For it would indeed be strange that amidst all the diversity of dimensions of heavenly bodies, it should invariably happen that the resultant movement of the combined masses should be reduced to such comparatively insignificant figures. But something more definite must yet be known in reference to the motion of the stars before this objection can be urged.

All that we are at present warranted to assume is simply that, of the comparatively few stars whose rate of motion has been properly measured, none have a greater velocity than 30 or 40 miles per second, while nothing whatever is known 
with certainty as to the rate of motion of the greater number of the stars.

There seems to be a somewhat prevailing misapprehension regarding the extent of our knowledge of stellar motions. Before we can ascertain the rate of motion of a star from its angular displacement of position in a given time, we must know its absolute distance. But it is only of the few stars which show a well-marked parallax that we can estimate the distance; for it is now generally admitted that there is no relation between the apparent magnitude and the real distance of a star. All that we know in regard to the distances of the greater mass of the stars is little else than mere conjecture. Even supposing we knew the absolute distance of a star and could measure its amount of displacement in a given time, still we could not be certain of its rate of motion unless we knew that it was moving directly at right angles to the line of vision, and not at the same time receding or advancing towards us; and this we could not determine by mere observation. The rate of motion, as determined from its observed change of position, may be, say, only twenty miles a second, while its actual velocity may be ten times that amount.

By spectrum-analysis it is true we can determine the rate at which a star may be advancing or receding along the line of sight independently of any knowledge of its distance. But this again does not give us the actual rate of motion, unless we are certain that it is moving directly to or from us. If it is at the same time moving transversely to the observer, its actual motion may be more than a hundred miles per second, while the rate at which it is receding or advancing, as determined by spectrum-analysis, may not be 20 miles a second. But in many cases it would be difficult to ascertain whether the star had a transverse motion or not. A star, for example, 1000 times more remote than $\alpha$ Centauri (that is, twenty thousand billion miles), though moving transversely to the observer at the enormous rate of 100 miles per second, would take upwards of 30 years to change its position so much as $1^{\prime \prime}$, and 1800 years to change its position $1^{\prime}$; in fact we should have to watch the star for a generation or two before we could be certain whether it was changing its position or not. And even after we had found with certainty that the star was shifting, and this at the rate of $1^{\prime}$ in 1800 years, we could not, without a knowledge of its distance, express the angle of displacement in miles. But from the apparent magnitude or brilliancy of the star, we could not determine whether its distance was 10 times, 100 times, or 1000 times that of a Centauri; and consequently we could form no con- 
jecture as to the actual velocity of the star. If we assumed its distance to be 10 times that of $\alpha$ Centauri, this would give a transverse velocity of one mile per second. If we assumed its distance to be 100 times that of $\alpha$ Centauri, this would give 10 miles a second as the velocity, and if 1000 times, the velocity of course would be 100 miles per second.

As there are but few of the stars which show a measurable parallax, and we have no other reliable method of estimating their distances*, it follows that in reference to the greater number of the stars, neither by spectrum-analysis nor by observation of their change of position can we determine their velocities. There does not, therefore, appear to be the shadow of a reason for believing that none of the stars has a motion of over 30 or 40 miles per second: for any thing that at present is known to the contrary, many of them may possess a proper motion enormously greater than that.

There is, however, an important point which seems to be overlooked in this objection, viz, that, unless the greater part of the motion of translation be transformed into heat, the chances are that no sun star will be formed. It is necessary to the formation of a sun which is to endure for millions of years, and to form the centre of a planetary system like our own, that the masses coming into collision should be converted into an incandescent nebulous mass. But the greater the amount of motion left unconverted into heat, the less is the chance of this condition being attained. A concussion which would leave the greater part of the motion of translation untransformed, would be likely as a general rule to produce merely a temporary star, which would blaze forth for a few years, or a few hundred years, or perhaps a few thousand years and then die out. In fact we have had several good examples of such since the time of Hipparchus. Now, although it may be true that, according to the law of chances, collisions producing temporary stars inust be far more numerous than those resulting in the formation of permanent stars, nevertheless the number of those temporary stars observable in the hearens may be perfectly insignificant in comparison with the number of permanent stars. Suppose there were as many as one hundred temporary stars formed for one permanent, and that on an average each should continue visible for 1000 years, there would not at the present moment be over half-a-dozen of such stars visible in the heavens.

4th. "Such collisions as the theory assumes are wholly

* It is true that we may one day be able to determine by spectrumanalysis the distance of some of the binary stars; but as yet this method has not been applied with sucress. 
hypothetical; it is extremely improbable that two cosmical bodies should move in the same straight line; and of two moving in different lines, it is improbable that either should impinge against the other." In reply, if there are stellar masses moving in all directions, collisions are unavoidable. It is true they will be of rare occurrence: but it is well that it is so ; for if they had been frequent the universe would be in a blaze, and its store of energy soon converted into heat.

II. On the Analysis of Alloys containing Copper, Zinc, and Nickel. By Thomas Bayrex, Assoc. R.C.Sc.I.*

7 THE analysis of these alloys can be very rapidly effected 1 by a combination of colorimetric and volumetric methods. The alloy is dissolved in nitric acid, and the solution then evaporated to dryness with excess of sulphuric acid to expel nitric acid, which must not be left in the solution.

Determination of the Copper.-The solution is mixed with excess of potassic iodide, which causes the formation of cuprous iodide, according to the following well-known reaction:-

$$
2 \mathrm{CuSO}_{4}+4 \mathrm{KI}=2 \mathrm{~K}_{2} \mathrm{SO}_{4}+\mathrm{Cu}_{2} \mathrm{I}_{2}+\mathrm{I}_{2} \text {. }
$$

The solution containing the precipitate is then titrated with a standard solution of sodic thiosulphate. The free iodine present is an exact measure of the copper, each gram of copper being equal to two grams of iodine. The following is the result of a series of determinations of copper made under various circumstances by this method:-

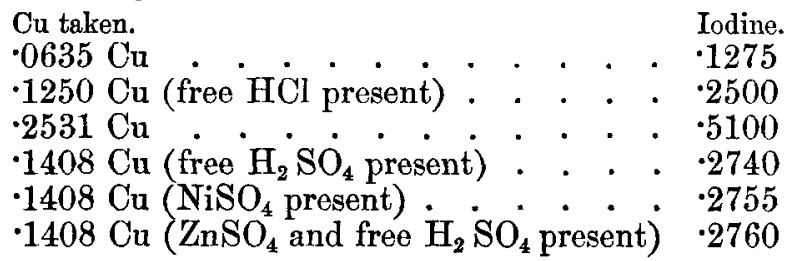

The solution, after the titration, is filtered and the precipitate washed. The filtrate is free from copper and contains the nickel and zinc. The absence of copper was proved in several experiments by evaporating to dryness and gently heating the residue, after which it was dissolved in a little dilute sulphuric acid and excess of ammonia added. In no instance was any blue colour perceptible.

Determination of the Nickel.-The fact will have been observed by chemists, that solutions of nickel and cobalt salts

* Communicated by the Author. 